\title{
On the Length and Area Regularization for Multiphase Level Set Segmentation
}

\author{
Luca Bertelli • Shivkumar Chandrasekaran . \\ Frédéric Gibou • B.S. Manjunath
}

Received: 27 September 2009 / Accepted: 23 April 2010 / Published online: 23 June 2010

(c) The Author(s) 2010. This article is published with open access at Springerlink.com

\begin{abstract}
In this paper we introduce novel regularization techniques for level set segmentation that target specifically the problem of multiphase segmentation. When the multiphase model is used to obtain a partitioning of the image in more than two regions, a new set of issues arise with respect to the single phase case in terms of regularization strategies. For example, if smoothing or shrinking each contour individually could be a good model in the single phase case, this is not necessarily true in the multiphase scenario.

In this paper, we address these issues designing enhanced length and area regularization terms, whose minimization yields evolution equations in which each level set function involved in the multiphase segmentation can "sense" the presence of the other level set functions and evolve accordingly. In other words, the coupling of the level set function, which before was limited to the data term (i.e. the proper segmentation driving force), is extended in a mathematically principled way to the regularization terms as well. The resulting regularization technique is more suitable to eliminate spurious regions and other kind of artifacts. An extensive experimental evaluation supports the model we introduce
\end{abstract}

\footnotetext{
L. Bertelli $(\bowtie) \cdot$ S. Chandrasekaran · B.S. Manjunath Department of Electrical and Computer Engineering, University of California, Santa Barbara, USA e-mail: lbertelli@ece.ucsb.edu

S. Chandrasekaran

e-mail: shiv@ece.ucsb.edu

B.S. Manjunath

e-mail:manj@ece.ucsb.edu

F. Gibou

Department of Computer Science and Department of Mechanical Engineering, University of California, Santa Barbara, USA

e-mail: fgibou@engineering.ucsb.edu
}

in this paper, showing improved segmentation performance with respect to traditional regularization techniques.

Keywords Image segmentation - Multiphase flows · Level set methods $\cdot$ Length regularization $\cdot$ Area regularization

\section{Introduction}

Image segmentation is one of the most studied problems in image analysis and many different approaches to segmentation have been introduced in the past. Among these, variational models based on the solution of partial differential equations (PDEs) have become increasingly popular in the last decade. They can be categorized into two different subgroups: region-based (Zhu et al. 1995; Zhu and Yuille 1996; Yezzi et al. 1999; Samson et al. 1999, 2000a, 2000b; Chan and Vese 2001; Tsai et al. 2001; Paragios and Deriche 1999, 2002; Rousson et al. 2003; Kim et al. 2005) and edge-based methods (Caselles et al. 1997; Kass et al. 1988; Malladi et al. 1994, 2004; Park and Keller 2001; Nguyen et al. 2003; Sapiro 1996, 1997; Kichenassamy et al. 1995; Goldenberg et al. 2001). Region-based approaches offer an advantage over edge-based segmentations in that they do not rely on edge detection, which can be sensitive to noise and clutter. Also, region-based algorithms are generally less dependent on initialization since they exploit global information of the image statistics.

A fundamental variational approach to region-based image segmentation was first presented by Mumford and Shah (1985), where the authors minimized a functional to approximate the image in a piecewise smooth way, penalizing at the same time the excessive length of the contours between regions. Later on, Chan and Vese minimized this functional within the level set framework (Osher and Sethian 1988), for 
both piecewise constant (Chan and Vese 2001) and smooth approximations of the image. In their work the authors represented two partitions as the positive and negative sub-level sets of a Lipschitz continuous function $\phi$ and they evolved $\phi$ in order to minimize the variance of each partition. Robustness to spurious noisy pixels is obtained by introducing two regularizing terms in the cost function, which penalize respectively the length of the contour (i.e. the zero level set of $\phi$ ) or the area of the region inside the contour. In summary the energy function to be minimized can be written as the sum of three different contributions:

$E=E_{d}+\mu E_{l}+v E_{a}$

where $E_{d}$ is the data term (i.e. the term which carries information about the image content), $E_{l}$ is the term penalizing the length and $E_{a}$ is the one penalizing the area, while $\mu$ and $v$ are two scalar weighting coefficients. Most of the literature in region-based segmentation targets the choice of the data term $E_{d}$ and several variants have been proposed, such as extensions to include higher order statistics (Dambreville et al. 2006; Kim et al. 2005; Rathi and Michailovich 2006), to cast the problem in a probabilistic framework (Paragios and Deriche 1999, 2002) or in a classification framework (Samson et al. 1999, 2000a, 2000b), to include additional information in the form of shape prior (Cremers et al. 2004, 2006; Cremers 2006; Raviv et al. 2005, 2006; Dambreville et al. 2006) (for other variants see Zhu and Yuille 1996; Yezzi et al. 1999; Aubert et al. 2003 and references therein). ${ }^{1}$

Much less attention has been devoted to improve the regularization terms $E_{l}$ and $E_{a}$. For example, the length of the contour can be replaced with a geodesic length with a metric based on the image gradient (Caselles et al. 1995, 1997; Paragios and Deriche 2002). This would penalize the presence of a contour more in a flat region than in correspondence of a high gradient region. For other contributions in this direction we refer the reader to Caselles et al. (1997), Paragios and Deriche (2002) and references therein. All these cited contributions targeted the binary segmentation case, i.e. the single level set function, also known as single phase case. When the multiphase model is used, a new set of issues arise that, to the best of our knowledge,

\footnotetext{
${ }^{1}$ Throughout this paper we adopt the data term introduced by Chan and Vese (2001) and then extended to the multiphase scenario in Vese and Chan (2002). This data-driven cost function, which aims at minimizing the variance of each partition, can be written as follows:

$E_{d}^{C V}=\sum_{i=1}^{N} \int_{\Omega}\left(u_{0}(\mathbf{x})-c_{i}\right)^{2} \chi_{i}(\mathbf{x}) d \mathbf{x}$

where $N$ is the number of regions/segments, $u_{0}$ is the original image, $c_{i}$ and $\chi_{i}$ for $i=1, \ldots, N$ are the means and the characteristic functions of each segment. In the 4-region case, (2) specializes in (43).
}

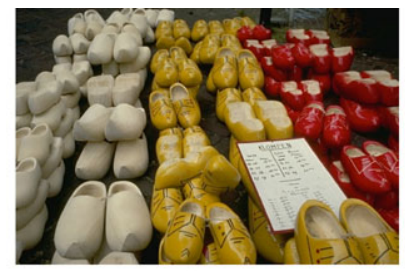

(a)

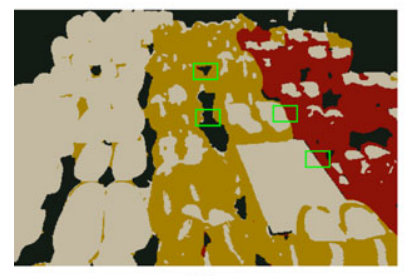

(b)
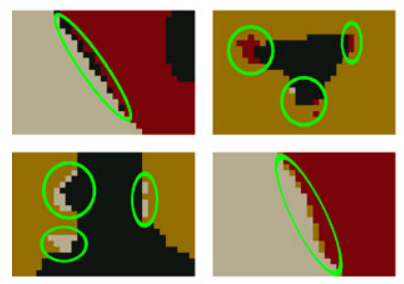

(d)

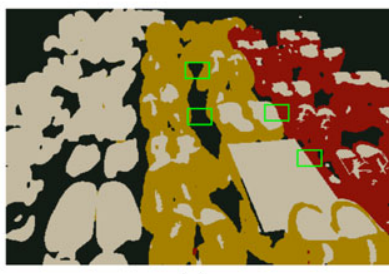

(c)
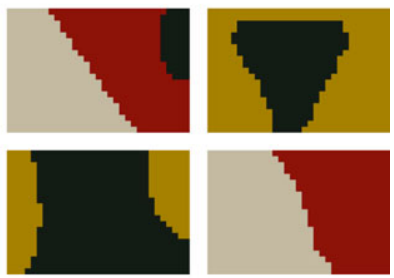

(e)
Fig. 1 (Color online) (a) Original image. (b, c, d, e) Comparison between the segmentations obtained using the traditional multiphase regularization approach (left column) and the proposed method (right column) - in both cases the data term is the one proposed by Vese and Chan (2002). In (b) and (c) we compare the 4 regions obtained using the two different regularization techniques. The areas within the green boxes are magnified in (d) and (e) to show that the spurious regions (highlighted within the green circles), present using the traditional regularization (d), are instead absent if the proposed method is applied (e)

have not been addressed in the literature. This paper can be considered a contribution in this direction.

Specifically, while smoothing contours can be a good regularization model in the single phase case to avoid the presence of boundaries wrapping around noisy spurious pixels, this does not necessarily hold true in the multiphase case. This problem becomes crucial in segmenting complex natural images (see Fig. 1 for example, where the segmentation is obtained using the 4-regions Chan-Vese model described in Vese and Chan 2002), where many of these spurious regions arise, particularly in correspondence of objects boundaries (that is where two level set functions can change sign simultaneously). The reason why traditional regularization based on smoothing is not enough to get rid of these spurious regions is explained pictorially in Fig. 2. Here two extremely smooth surfaces are running parallel and spatially close to each other. The multiphase segmentation model can produce a spurious region located between the two boundaries. The traditional length and area regularization terms, which act on each contour independently, would not get rid of this spurious region since the two contours are already 
Fig. 2 (a) Two level set functions running parallel and close to each other. The two black lines are the zero level sets. (b) Projection of the zero level sets on the image plane, showing the resulting multi-phase segmentation. Note the presence of a small spurious region between the two interfaces. Traditional regularization techniques, based on smoothing (i.e. length regularization) or shrinking (i.e. area regularization) each contour independently from the other contours, are not suitable for getting rid of this region



(a)

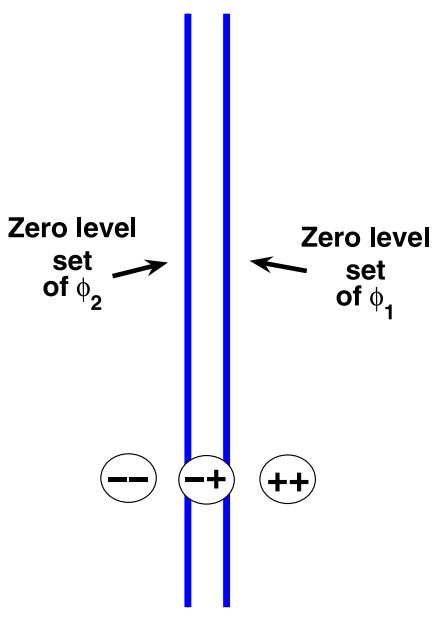

(b) sufficiently smooth. Therefore there is a need to rethink the regularization terms in a multiphase perspective, introducing the possibility for each contour to "sense" the presence of the other contours and evolve accordingly.

The main contributions of this paper are targeting these issues and can be summarized as follows:

- Formulation of a length regularization term $E_{l}$, in which smoothing happens selectively, depending on the reciprocal position of the contours.

- Formulation of an area regularization term $E_{a}$, in which shrinking/expanding is conditioned on the presence of other contours, providing a more principled regularization effect.

- Extensive experimental validation of the proposed regularization models.

This paper is organized as follows. In Sect. 2 we briefly review regularization techniques in presence of a single level set function, while Sect. 3 extends the concepts to the multiphase scenario. In particular, Sect. 3.1 deals with length regularization, while Sect. 3.2 is concerned with area regularization. After presenting experimental results in Sect. 4, we briefly conclude in Sect. 5 .

\section{Single Phase Length and Area Regularizations}

In single phase level set segmentation a curve $C$, the boundary of an open set $R_{i}(C) \in \Omega$ (i.e. $C=\partial R_{i}(C)$ ), is implicitly represented as the zero level set of a Lipschitz function $\phi: \Omega \mapsto \mathbb{R}$ (Osher and Sethian 1988). The function $\phi$ is positive for the points within the set $R_{i}(C)$ and negative elsewhere (i.e. for the points within $R_{o}(C)=\Omega \backslash R_{i}(C)$ ). Therefore the Heaviside function $H(\phi)$, along with its complementary $(1-H(\phi))$, can serve as an indicator function for the points in $R_{i}(C)$ and $R_{o}(C)$ respectively (Chan and Vese 2001):

$\chi_{1}=H(\phi)= \begin{cases}1 & \text { if } \phi>0 \\ 0 & \text { elsewhere }\end{cases}$

$\chi_{2}=(1-H(\phi))= \begin{cases}1 & \text { if } \phi<0 \\ 0 & \text { elsewhere }\end{cases}$

As mentioned in the introduction, the length regularization term is expressed as a term proportional to the length of the contour (Chan and Vese 2001). Translating this concept using a formal notation we can write:

$$
\begin{aligned}
E_{l 1} & =\int_{\Omega}\left|\nabla \chi_{1}(\mathbf{x})\right| d \mathbf{x}=\int_{\Omega}\left|\nabla \chi_{2}(\mathbf{x})\right| d \mathbf{x} \\
& =\int_{\Omega}|\nabla H(\phi(\mathbf{x}))| d \mathbf{x}=\int_{\Omega} \delta(\phi(\mathbf{x}))|\nabla \phi(\mathbf{x})| d \mathbf{x}
\end{aligned}
$$

In order to compute the Euler-Lagrange equation for the function $\phi$ such that the penalty term is minimized, we need to consider slight regularizations of the functions $H$ and $\delta$. We denote these regularized functions as $H_{\epsilon}$ and $\delta_{\epsilon}$, and from now on we will proceed informally using them in place of the non-regularized ones. For a formal proof of existence of minimizers for the non-regularized case (i.e. $\epsilon=0$ ) we refer the reader to Appendix A, while an extensive analysis of the behavior in the limit (i.e. as $\epsilon \rightarrow 0$ ) is presented in Appendix B. Minimizing (5) using calculus of variations, we obtain the PDE that evolves the level sets in the proximity of the zero level set under a motion by mean curvature, enforcing the smoothness of the contour:

$$
\frac{\partial \phi(\mathbf{x})}{\partial t}=\delta_{\epsilon}(\phi(\mathbf{x})) \operatorname{div}\left(\frac{\nabla \phi(\mathbf{x})}{|\nabla \phi(\mathbf{x})|}\right)
$$

As mentioned in the introduction, a second regularization term can be expressed in terms of the area of the region in- 
side of the contour (i.e. $\chi_{1}$ ) (Chan and Vese 2001). Using the same notation as above, we can write this term as:

$E_{a 1}=\int_{\Omega} \chi_{1}(\mathbf{x}) d \mathbf{x}=\int_{\Omega} H(\phi(\mathbf{x})) d \mathbf{x}$

Minimizing using calculus of variations yields the PDE which shrinks the contour, getting rid in this way of small spurious regions:

$\frac{\partial \phi(\mathbf{x})}{\partial t}=-\delta_{\epsilon}(\phi(\mathbf{x}))$

The use of the flows in (8) and (6), along with the one that minimizes the data term, are perfectly suitable in the case of binary segmentation (i.e. background/foreground segmentation using only one level set function). On the other hand, in the following section we will demonstrate that, for the multiregion segmentation case, a reformulation of the traditional regularization term is needed in order to achieve accurate and reliable results.

\section{Multiphase Length and Area Regularizations}

The use of only one level set function $\phi$, discussed in the previous section, can provide a separation of the image into only two segments, corresponding respectively to the positive and negative sub-level sets of $\phi$. In Vese and Chan (2002) the authors showed how $K$ level set functions can be used to construct up to $N=2^{K}$ different indicator functions and therefore to represent up to $N$ different regions. Using this convention, in the case of $N=4$ regions, we can write the four characteristic functions as:

$$
\begin{aligned}
\chi_{1} & =H_{\epsilon}\left(\phi_{1}\right) H_{\epsilon}\left(\phi_{2}\right)= \begin{cases}1 & \text { if } \phi_{1}>0 \text { and } \phi_{2}>0 \\
0 & \text { elsewhere }\end{cases} \\
\chi_{2} & =H_{\epsilon}\left(\phi_{1}\right)\left(1-H_{\epsilon}\left(\phi_{2}\right)\right)= \begin{cases}1 & \text { if } \phi_{1}>0 \text { and } \phi_{2}<0 \\
0 & \text { elsewhere }\end{cases} \\
\chi_{3} & =\left(1-H_{\epsilon}\left(\phi_{1}\right)\right) H_{\epsilon}\left(\phi_{2}\right)= \begin{cases}1 & \text { if } \phi_{1}<0 \text { and } \phi_{2}>0 \\
0 & \text { elsewhere }\end{cases} \\
\chi_{4} & =\left(1-H_{\epsilon}\left(\phi_{1}\right)\right)\left(1-H_{\epsilon}\left(\phi_{2}\right)\right) \\
& = \begin{cases}1 & \text { if } \phi_{1}<0 \text { and } \phi_{2}<0 \\
0 & \text { elsewhere }\end{cases}
\end{aligned}
$$

In the general case, the $N$ indicator functions $\chi_{i}$ with $i=$ $1, \ldots, N$ are given by all the possible sign combinations of the $K$ level set functions $\phi_{j}$, with $j=1, \ldots, K$.

In the following we design novel multiphase regularization terms, such that, during the regularization process, the contours could sense the presence of the other nearby contours and evolve accordingly. In particular we require the regularization term (i.e. combination of area and length regularization) to satisfy the following two properties:

1. the effect of the regularization must not separate two overlapping contours,

2. two contours running close to each other must either snap onto each other or move apart from each other, due to the regularization term.

This section is composed of two parts: Sect. 3.1 concerning the length term and Sect. 3.2 concerning the area term. In each part, for the sake of clarity and readability, we will start discussing about the 4 regions case (i.e. two level set functions $\phi_{1}$ and $\phi_{2}$ ) and then we will generalize our model to the $N$ regions case.

\subsection{Length Regularization}

\subsubsection{Four Regions Case}

In the 4 regions case, the ideal length regularization term (i.e. the effective total length of the contour) can be written as:

$E_{l 2}=\frac{1}{2} \sum_{i=1}^{4} \int_{\Omega}\left|\nabla \chi_{i}(\mathbf{x})\right| d \mathbf{x}$

Unfortunately the minimization of this term using calculus of variation leads to an extremely complicated expression, due to the coupling of the two level set functions $\phi$ 's, not easily implementable. The solution provided by Vese and Chan (2002) consists in a simplification of the problem, decoupling the dependency of the length term upon the two level set functions. The simplified term they introduced is the following:

$$
\begin{aligned}
E_{l 2} & =\sum_{i=1}^{2} \int_{\Omega}\left|\nabla H_{\epsilon}\left(\phi_{i}(\mathbf{x})\right)\right| d \mathbf{x} \\
& =\sum_{i=1}^{2} \int_{\Omega} \delta_{\epsilon}\left(\phi_{i}(\mathbf{x})\right)\left|\nabla \phi_{i}(\mathbf{x})\right| d \mathbf{x}
\end{aligned}
$$

The main drawback of this model consists in the fact that some parts of the contours (i.e. the overlapping parts) are counted twice (see Fig. 3). Therefore nothing guarantees that two overlapping contours will not separate during the evolution process, since separating or merging contours does not change the regularization cost function in use. This can potentially violate Property 1 stated at the beginning of the section.

In order to always enforce this property, we need to redesign the length term, making sure that it represents the effective total length of the contour (i.e. without counting any 


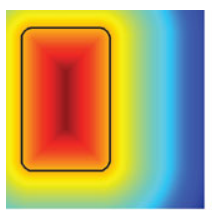

(a)

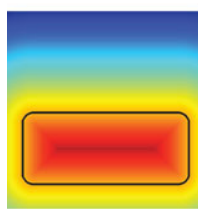

(e)

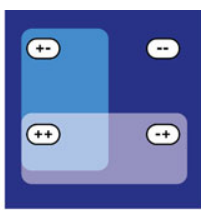

(b)

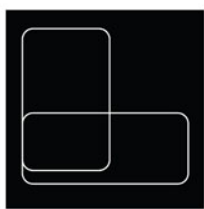

(f)

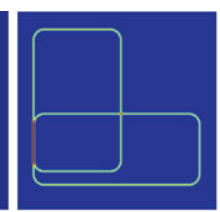

(c)

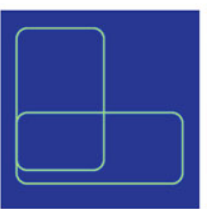

(g)

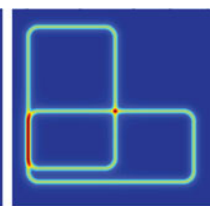

(d)

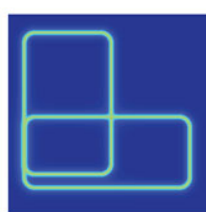

(h)
Fig. 3 (a) and (e) Two level set functions $\phi_{1}$ and $\phi_{2}$ (the zero level set is marked in black). (b) The correspondent segmentation in four regions. (f) The ground truth boundaries. (c) and (d) Regularization using (10) for $\epsilon=1$ and $\epsilon=5$ respectively. Notice that the overlapping parts are counted twice. (g) and (h) Regularization using (11) for $\epsilon=1$ and $\epsilon=5$ respectively

part twice) as in (10), but with a more approachable formulation than the one in (9). The solution we propose consists in subtracting from (9) a term which compensates for those segments where two contours are overlapping.

$$
\begin{aligned}
E_{l 2}= & \sum_{i=1}^{2} \int_{\Omega}\left|\nabla H_{\epsilon}\left(\phi_{i}(\mathbf{x})\right)\right| d \mathbf{x} \\
& -\gamma \int_{\Omega}\left|\nabla H_{\epsilon}\left(\phi_{1}(\mathbf{x})\right)\right|\left|\nabla H_{\epsilon}\left(\phi_{2}(\mathbf{x})\right)\right| d \mathbf{x} \\
= & \sum_{i=1}^{2} \int_{\Omega} \delta_{\epsilon}\left(\phi_{i}(\mathbf{x})\right)\left|\nabla \phi_{i}(\mathbf{x})\right| d \mathbf{x} \\
& -\gamma \int_{\Omega} \delta_{\epsilon}\left(\phi_{1}(\mathbf{x})\right)\left|\nabla \phi_{1}(\mathbf{x})\right| \delta_{\epsilon}\left(\phi_{2}(\mathbf{x})\right)\left|\nabla \phi_{2}(\mathbf{x})\right| d \mathbf{x}
\end{aligned}
$$

where $\gamma$ is a constant coefficient. If the point $\mathbf{x}_{0}$ belongs to the zero level set of only one of the two level set functions $\phi_{i}$ 's (let us assume w.l.o.g. that is on the zero level set of $\left.\phi_{1}\right)$, we have that:

$\delta_{\epsilon}\left(\phi_{1}\left(\mathbf{x}_{0}\right)\right)=\delta_{\epsilon}(0)$

$\delta_{\epsilon}\left(\phi_{2}\left(\mathbf{x}_{0}\right)\right) \approx 0$

$\delta_{\epsilon}\left(\phi_{1}\left(\mathbf{x}_{0}\right)\right) \delta_{\epsilon}\left(\phi_{2}\left(\mathbf{x}_{0}\right)\right) \approx 0$

Assuming the $\phi_{i}$ 's being signed distance functions (i.e. $\left.\left|\nabla \phi_{i}(\mathbf{x})\right|=1 \forall \mathbf{x} \in \Omega\right)$, the contribution of the point $\mathbf{x}_{0}$ to the whole integral is therefore $\delta_{\epsilon}(0)$. We want to choose the constant coefficient $\gamma$, such that the contribution of a point $\mathbf{x}_{1}$, which belongs to the zero level set of both $\phi_{i}$ 's, is still $\delta_{\epsilon}(0)$ (note that, if $\mathbf{x}_{1}$ does not belong to both zero level sets, the term that multiplies $\gamma$ in (11) vanishes and hence the value of $\gamma$ is irrelevant at those locations). This will prevent from counting $\mathbf{x}_{1}$ twice, as it happens with the cost function by Vese and Chan in (10). By simple algebra we obtain:

$\gamma=\frac{1}{\delta_{\epsilon}(0)}$

For a more formal explanation regarding the choice of this normalization coefficient $\gamma$ we refer the reader to Appendix B. Minimizing (11) w.r.t. $\phi_{1}$ (or equivalently $\phi_{2}$ ) using calculus of variations and parameterizing the descent via a virtual time variable $t$, we obtain the following PDE, which evolves $\phi_{1}$ in the direction of steepest descent of the cost function:

$$
\begin{aligned}
\frac{\partial \phi_{1}(\mathbf{x})}{\partial t}= & \delta_{\epsilon}\left(\phi_{1}(\mathbf{x})\right)\left[1-\frac{1}{\delta_{\epsilon}(0)} \delta_{\epsilon}\left(\phi_{2}(\mathbf{x})\right)\left|\nabla \phi_{2}(\mathbf{x})\right|\right] \\
& \times \operatorname{div}\left(\frac{\nabla \phi_{1}(\mathbf{x})}{\left|\nabla \phi_{1}(\mathbf{x})\right|}\right)
\end{aligned}
$$

The term within the square brackets can be viewed as an adaptive coefficient for the curvature term. In the case of the regularizing term proposed by Vese and Chan (10), this coefficient is constant equal to 1 , providing a smoothing effect which does not sense the presence of the other level set function $\phi_{2}$. On the other hand, in the proposed model the coefficients depends upon $\phi_{2}$ and therefore provides a smoothing coupled with the information provided by $\phi_{2}$.

Since $\delta_{\epsilon}(z)$ is a function which attains its maximum for $z=0$, the term within the square brackets is always greater than or equal to zero. This prevents the flow from becoming a negative motion by mean curvature and therefore retains the well-posedness of the PDE (13). We will show that this is true not only for $K=2$ (i.e. two level set functions), but for any choice of $K \geq 2$.

In addition, expressing the cost in terms of the effective length of the contour (i.e. making sure that the contribution of each point belonging to at least one contour is always $\left.\delta_{\epsilon}(0)\right)$ has the consequence of enforcing Property 1 . In fact, if two contours are overlapping at a particular location $\mathbf{x}$, it is straightforward to see that the term within the square brackets in (13) vanishes, preventing the regularization effect from separating the contours. We visually demonstrate this property in Fig. 4.

An attempt to solve the problem of designing a cost function that truly represents the length of the multiphase contour is briefly described by Vese (2003). In this paper the author modifies (10), to take into account the parts of the contour counted twice, as follows:

$$
\begin{aligned}
E_{l 2}^{\#}= & \int_{\Omega}\left|\nabla H\left(\phi_{1}(\mathbf{x})\right)\right| d \mathbf{x} \\
& +\int_{\Omega}\left|\nabla H\left(\phi_{2}(\mathbf{x})\right)\right|\left(2-H\left(\phi_{1}(\mathbf{x})\right)\right. \\
& \left.-H\left(-\phi_{1}(\mathbf{x})\right)\right) d \mathbf{x}
\end{aligned}
$$




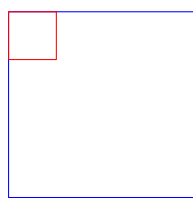

(a)

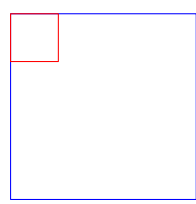

(e)

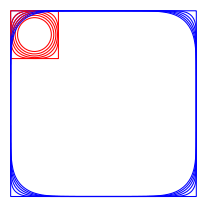

(b)

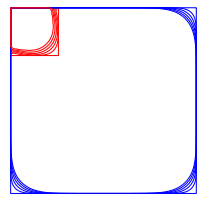

(f)

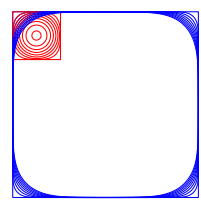

(c)

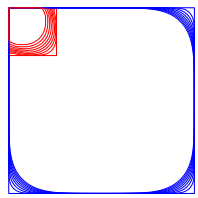

(g)

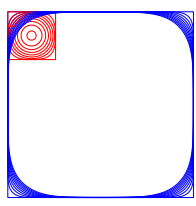

(d)

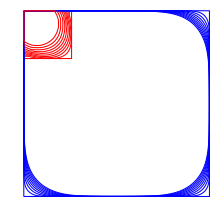

(h)
Fig. 4 First row: Evolution using the traditional length term (10). (a) Initial configuration, (b, c) Two stages of the evolution. Second row: Evolution using the proposed length term (11). (e) Initial configuration, (f, g) Two stages of the evolution. Note that the overlapping parts of the contours are not separated by the proposed regularization, while that is not the case if the traditional regularization is used

There are two main drawbacks which differentiate this solution from the one proposed in this paper. First of all (14) requires the function $H$ to be the ideal step function (in particular, $H$ needs to be defined continuous from the right, that is $H(0)=1)$. In numerical implementation, $H$ is usually regularized such that $H(z)=1-H(-z)$, which would make the term $\left(2-H\left(\phi_{1}(\mathbf{x})\right)-H\left(-\phi_{1}(\mathbf{x})\right)\right)$ in (14) constantly equal to 1 and therefore ineffective. On the other hand, the model proposed in this paper maintains its properties also upon regularization of the step functions. In addition, the term in (14) is not symmetric with respect to $\phi_{1}$ and $\phi_{2}$, which means that the evolution equation of $\phi_{1}$ will differ from the one of $\phi_{2}$. This desirable symmetry property is instead achieved by the proposed term, see (11).

\subsubsection{N Regions Case}

In the general case, i.e. in the case of $N$ regions represented via $K$ level set functions, we construct the $K$-component vector:

$$
\begin{aligned}
\Psi(\mathbf{x})= & {\left[\left|\nabla H_{\epsilon}\left(\phi_{1}(\mathbf{x})\right)\right|,\left|\nabla H_{\epsilon}\left(\phi_{2}(\mathbf{x})\right)\right|, \ldots,\right.} \\
& \left.\left|\nabla H_{\epsilon}\left(\phi_{K}(\mathbf{x})\right)\right|\right]^{T}
\end{aligned}
$$

Denoting $\mathbf{e}_{i}$ the $K$-dimensional indicator vector, which has all zeros except a one at index $i$, we can write the general expression for the regularization cost function in differential form as:

$$
\begin{aligned}
e_{l K}(\mathbf{x})= & \sum_{i=1}^{K} \mathbf{e}_{i}^{T} \Psi(\mathbf{x}) \\
& +\frac{-1}{\delta_{\epsilon}(0)} \sum_{i_{1}=1}^{K-1} \sum_{i_{2}=i_{1}+1}^{K}\left(\mathbf{e}_{i_{1}}^{T} \Psi(\mathbf{x})\right)\left(\mathbf{e}_{i_{2}}^{T} \Psi(\mathbf{x})\right)
\end{aligned}
$$

$$
\begin{aligned}
& +\left(\frac{-1}{\delta_{\epsilon}(0)}\right)^{2} \sum_{i_{1}=1}^{K-2} \sum_{i_{2}=i_{1}+1}^{K-1} \sum_{i_{3}=i_{2}+1}^{K}\left(\mathbf{e}_{i_{1}}^{T} \Psi(\mathbf{x})\right) \\
& \times\left(\mathbf{e}_{i_{2}}^{T} \Psi(\mathbf{x})\right)\left(\mathbf{e}_{i_{3}}^{T} \Psi(\mathbf{x})\right) \\
& +\cdots \\
& +\left(\frac{-1}{\delta_{\epsilon}(0)}\right)^{K-2} \sum_{i_{1}=1}^{2} \ldots \sum_{i_{K}=i_{K-1}+1}^{K}\left(\mathbf{e}_{i_{1}}^{T} \Psi(\mathbf{x})\right) \ldots \\
& \times\left(\mathbf{e}_{i_{K}}^{T} \Psi(\mathbf{x})\right) \\
& +\left(\frac{-1}{\delta_{\epsilon}(0)}\right)^{K-1}\left(\mathbf{e}_{1}^{T} \Psi(\mathbf{x})\right) \ldots\left(\mathbf{e}_{K}^{T} \Psi(\mathbf{x})\right)
\end{aligned}
$$

The integral form of the regularization term is then obtained integrating $e_{l K}$ over the domain:

$E_{l K}=\int_{\Omega} e_{l K}(\mathbf{x}) d \mathbf{x}$

With the following lemma, we prove that the value of (16) represents the effective length of the contour (i.e. no parts are counted twice). As explained for the 4 regions case, this will ensure that Property 1 is always satisfied.

Lemma 1 For any point $\mathbf{x}_{\mathbf{0}} \in \Omega$ belonging to the zero level set of $n$ level set functions $(1 \leq n \leq K)$, the value of $e_{l K}\left(\mathbf{x}_{0}\right)$ is approximately $\delta_{\epsilon}(0)$, regardless the value of $n$.

Proof In this case, in fact, the vector $\Psi\left(\mathbf{x}_{\mathbf{0}}\right)$ has $n$ entries equal to $\delta_{\epsilon}(0)$ and the rest of the entries are approximately zero. We can therefore re-write (15) as:

$$
\begin{aligned}
e_{l K}\left(\mathbf{x}_{0}\right)= & \delta_{\epsilon}(0)\left(\begin{array}{l}
n \\
1
\end{array}\right) \\
& +\frac{-1}{\delta_{\epsilon}(0)}\left(\delta_{\epsilon}(0)\right)^{2}\left(\begin{array}{l}
n \\
2
\end{array}\right) \\
& +\left(\frac{1}{\delta_{\epsilon}(0)}\right)^{2}\left(\delta_{\epsilon}(0)\right)^{3}\left(\begin{array}{l}
n \\
3
\end{array}\right) \\
& +\cdots \\
& +\left(\frac{-1}{\delta_{\epsilon}(0)}\right)^{K-1}\left(\delta_{\epsilon}(0)\right)^{K}\left(\begin{array}{l}
n \\
K
\end{array}\right)
\end{aligned}
$$

Therefore we have that: ${ }^{2}$

$$
\begin{aligned}
e_{l K}\left(\mathbf{x}_{0}\right) & =\delta_{\epsilon}(0) \sum_{k=1}^{K}(-1)^{k-1}\left(\begin{array}{l}
n \\
k
\end{array}\right) \\
& =\delta_{\epsilon}(0) \sum_{k=1}^{n}(-1)^{k-1}\left(\begin{array}{l}
n \\
k
\end{array}\right) \\
& =-\delta_{\epsilon}(0)\left[\sum_{k=0}^{n}(-1)^{k}\left(\begin{array}{l}
n \\
k
\end{array}\right)-1\right]
\end{aligned}
$$

${ }^{2}$ Note that $\left(\begin{array}{l}n \\ k\end{array}\right)=0$ if $k>n$. 
Now from a basic property of the binomial coefficient we have:

$(x+y)^{n}=\sum_{k=0}^{n}\left(\begin{array}{l}n \\ k\end{array}\right) x^{n-k} y^{k}$

which implies for $x=1$ and $y=-1$ :

$\sum_{k=0}^{n}(-1)^{k}\left(\begin{array}{l}n \\ k\end{array}\right)=0$

Substituting (20) into (18) concludes the proof.

Minimizing (16) w.r.t. $\phi_{1}$ (the derivation for the other $\phi_{i}$, with $i=2, \ldots, K$, is identical) using calculus of variations and parameterizing the descent, as done before, via the variable $t$, we obtain the following evolution equation for $\phi_{1}$ :

$$
\begin{aligned}
\frac{\partial \phi_{1}(\mathbf{x})}{\partial t} & \\
= & \delta_{\epsilon}\left(\phi_{1}(\mathbf{x})\right)\left[1+\frac{-1}{\delta_{\epsilon}(0)} \sum_{i_{2}=2}^{K}\left(\mathbf{e}_{i_{2}}^{T} \Psi(\mathbf{x})\right)\right. \\
& +\left(\frac{-1}{\delta_{\epsilon}(0)}\right)^{2} \sum_{i_{2}=2}^{K-1} \sum_{i_{3}=i_{2}+1}^{K}\left(\mathbf{e}_{i_{2}}^{T} \Psi(\mathbf{x})\right)\left(\mathbf{e}_{i_{3}}^{T} \Psi(\mathbf{x})\right) \\
& +\cdots \\
& +\left(\frac{-1}{\delta_{\epsilon}(0)}\right)^{K-2} \sum_{i_{2}=2}^{3} \ldots \sum_{i_{K}=i_{K-1}+1}^{K}\left(\mathbf{e}_{i_{2}}^{T} \Psi(\mathbf{x})\right) \ldots \\
& \times\left(\mathbf{e}_{i_{K}}^{T} \Psi(\mathbf{x})\right) \\
& \left.+\left(\frac{-1}{\delta_{\epsilon}(0)}\right)^{K-1}\left(\mathbf{e}_{2}^{T} \Psi(\mathbf{x})\right) \ldots\left(\mathbf{e}_{K}^{T} \Psi(\mathbf{x})\right)\right] \\
& \times \operatorname{div}\left(\frac{\nabla \phi_{1}(\mathbf{x})}{\left|\nabla \phi_{1}(\mathbf{x})\right|}\right)
\end{aligned}
$$

To guarantee the well-posedness of the PDE (21), we need to show that the quantity within the square brackets is always greater than or equal to zero for any point in the domain. In order to accomplish this, we need the following Lemma.

Lemma 2 Let $K \in \mathbb{N}$ and let $y \in \mathbb{R}^{K}$. Moreover, let $y_{i}$ denote the $i$-th component of $y$. Assume that $0 \leq y_{i} \leq 1$, for $i=1, \ldots, K$. Define the quantity

$$
\begin{aligned}
f_{K}(\mathbf{y})= & 1-\sum_{i_{2}=2}^{K} y_{i_{2}}+\sum_{i_{2}=2}^{K-1} \sum_{i_{3}=i_{2}+1}^{K} y_{i_{2}} y_{i_{3}}+\cdots \\
& +(-1)^{K-2} \sum_{i_{2}=2}^{3} \ldots \sum_{i_{K}=i_{K-1}+1}^{K} y_{i_{2}} \ldots y_{i_{K}} \\
& +(-1)^{K-1} y_{2} \ldots y_{K}
\end{aligned}
$$

Then

$$
f_{K}(\mathbf{y}) \geq 0
$$

Proof We will proceed by induction. In the case of $K=2$ we have:

$$
f_{2}(\mathbf{y})=1-y_{2}
$$

which is greater than or equal to zero, since by definition $0 \leq y_{2} \leq 1$. We now show that for $K=m$, we have that $f_{m}(\mathbf{y}) \geq 0$ if we assume that $f_{m-1}(\mathbf{y}) \geq 0$. In fact, $f_{m}(\mathbf{y})$ can be re-written in the following way:

$$
\begin{aligned}
f_{m}(\mathbf{y})=1 & -\left(y_{m}+\sum_{i_{2}=2}^{m-1} y_{i_{2}}\right) \\
& +\left(y_{m} \sum_{i_{2}=2}^{m-1} y_{i_{2}}+\sum_{i_{2}=2}^{m-2} \sum_{i_{3}=i_{2}+1}^{m-1} y_{i_{2}} y_{i_{3}}\right)+\ldots \\
& +(-1)^{m-2}\left(y_{m} \sum_{i_{2}=2}^{3} \ldots \sum_{i_{m-1}=i_{m-2}+1}^{m-1} y_{i_{2}} \ldots y_{i_{m-1}}\right. \\
& \left.+y_{2} \ldots y_{m-1}\right)+(-1)^{m-1} y_{m} y_{2} \ldots y_{m-1}
\end{aligned}
$$

Now separating the terms where $y_{m}$ is present from the other ones, one can see that:

$$
f_{m}(\mathbf{y})=\left(1-y_{m}\right) f_{m-1}(\mathbf{y})
$$

Since $0 \leq y_{m} \leq 1$ by definition and $f_{m-1}(\mathbf{y}) \geq 0$ by induction hypothesis, we conclude that also $f_{m}(\mathbf{y}) \geq 0$.

The following result is a consequence of the above Lemma.

Corollary 1 The quantity within the square brackets in (21), is always greater than or equal to 0 for any $x \in \Omega$.

\section{Proof Define}

$\bar{\Psi}(\mathbf{x}):=\frac{1}{\delta_{\epsilon}(0)} \Psi(\mathbf{x})$

Hence the quantity within square bracket in (21), can be rewritten as:

$$
\begin{aligned}
1 & -\sum_{i_{2}=2}^{K} \bar{\Psi}_{i_{2}}+\sum_{i_{2}=2}^{K-1} \sum_{i_{3}=i_{2}+1}^{K} \bar{\Psi}_{i_{2}} \bar{\Psi}_{i_{3}}+\cdots \\
& +(-1)^{K-2} \sum_{i_{2}=2}^{3} \ldots \sum_{i_{K}=i_{K-1}+1}^{K} \bar{\Psi}_{i_{2}} \ldots \bar{\Psi}_{i_{K}} \\
& +(-1)^{K-1} \bar{\Psi}_{2} \ldots \bar{\Psi}_{K}
\end{aligned}
$$


where we dropped the dependency of $\bar{\Psi}$ upon $\mathbf{x}$ for the sake of clarity. Since, $\bar{\Psi}(\mathbf{x})$ is the normalized version of $\Phi(\mathbf{x})$ by $\delta_{\epsilon}(0)$, we have that $0 \leq \bar{\Psi}_{i}(\mathbf{x}) \leq 1$, for $i=1, \ldots, K$. Then the result follows from Lemma 2.

\subsection{Area Regularization}

\subsubsection{Four Regions Case}

For the 4 regions case, similarly to what has been done for the length in (10), a naive version of the area regularization term can be written as:

$E_{a 2}=\sum_{i=1}^{2} \int_{\Omega} H_{\epsilon}\left(\phi_{i}(\mathbf{x})\right) d \mathbf{x}$

Minimizing this term leads to a shrinking force that acts independently on each level set function $\phi_{i}$. The lack of a term which couples the two level set functions prevents them from sensing each other's position during the evolution. Reasoning along the lines of what we have done for the length term, we modify (28) by considering that it does not faithfully represent the area of the partitions. In fact the area term as defined in (28) penalizes three partitions out of four (i.e. all the partitions for which at least one level set function is positive). In addition, the partition where both $\phi_{1}$ and $\phi_{2}$ are positive is penalized twice as much as the others.

The idea we apply to modify this term is to penalize only half of the partitions. In particular, we introduce a coupling term such that only partitions with only one level set function being positive are penalized:

$$
\begin{aligned}
E_{a 2}= & \sum_{i=1}^{2} \int_{\Omega} H_{\epsilon}\left(\phi_{i}(\mathbf{x})\right) d \mathbf{x} \\
& -2 \int_{\Omega} H_{\epsilon}\left(\phi_{1}(\mathbf{x})\right) H_{\epsilon}\left(\phi_{2}(\mathbf{x})\right) d \mathbf{x}
\end{aligned}
$$

The PDE corresponding to the steepest descent minimization of (29) with respect to $\phi_{1}$ is:

$$
\frac{\partial \phi_{1}(\mathbf{x})}{\partial t}=-\delta_{\epsilon}\left(\phi_{1}(\mathbf{x})\right)\left[1-2 H_{\epsilon}\left(\phi_{2}(\mathbf{x})\right)\right]
$$

The evolution equation for $\phi_{2}$ is obtained interchanging $\phi_{1}$ and $\phi_{2}$ in (30). The coefficient within the square brackets is important since it forces two contours spatially close to each other to snap onto one another or to move away from each other, preventing the formation of contours running close and parallel to each other. For example, let us assume that $\phi_{1}$ and $\phi_{2}$ are concentric, with $\phi_{2}$ laying inside the region where $\phi_{1}$ is positive. The term within the

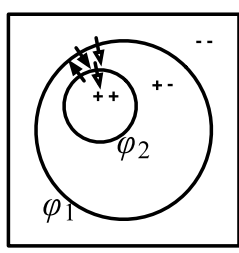

(a)

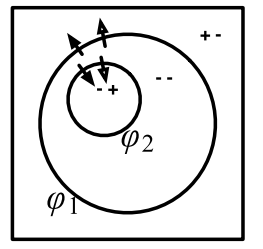

(c)

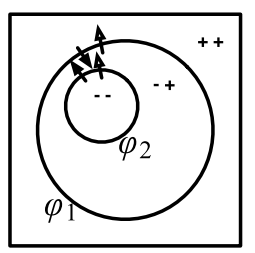

(b)

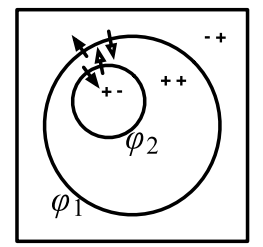

(d)
Fig. 5 Effect of the evolution under the area regularization force, for all the possible combinations of signs of two concentric level set functions. Solid arrows: proposed area regularization. Empty arrows: standard area regularization (Vese and Chan 2002)

square brackets will be positive for the PDE regulating the evolution of $\phi_{1}$, forcing its contour to shrink, while negative in the case of $\phi_{2}$, forcing its contour to expand. The resulting coupled evolution will see the zero level set of $\phi_{1}$ shrinking and snapping onto the expanding zero level set of $\phi_{2}$. Other possible situations are pictorially represented in Fig. 5 and compared with the regularization using the standard area term. With the standard regularization, for half of the possible configurations, the two contours either both shrink or expand. This does not contribute to removing contours that are close and parallel to each other, and in turn would yield non-desirable double edges in a segmentation process. On the other hand, using the proposed area regularization, every possible configuration corresponds to a situation in which two contours spatially close are forced to either snap onto one another or move away from each other. Therefore this term guarantees that Property 2 is always satisfied. In addition, if $\mathbf{x}$ belongs to both contours, the term within the square brackets in (30) vanishes, (since $\left.H_{\epsilon}(0)=1 / 2\right)$. This guarantees that Property 1 is always satisfied as well. In Fig. 6, we show the evolution of two level set functions under the traditional and the proposed regularization term.

\subsubsection{N Regions Case}

In the case of $N$ regions represented via $K$ level set functions, we can write the area-penalizing cost function as:

$E_{a K}(\mathbf{x})=\int_{\Omega} e_{a K}(\mathbf{x}) d \mathbf{x}$ 


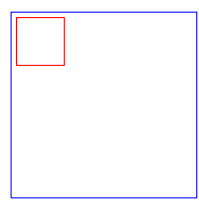

(a)



(e)

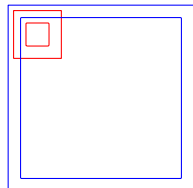

(b)

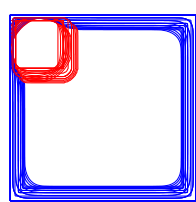

(f)

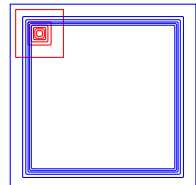

(c)

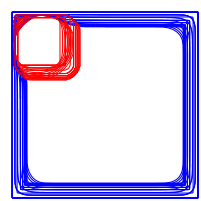

(g)

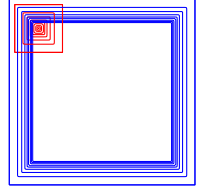

(d)

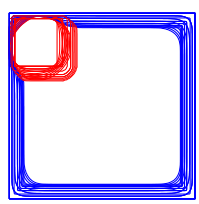

(h)
Fig. 6 First row: Level set evolution using the traditional area term (a) Initial configuration, $(\mathbf{b}, \mathbf{c})$ Two stages of the evolution. Second row: Evolution using the proposed area term, (e) Initial configuration, (f, $\mathbf{g})$ Two stages of the evolution. Note that using the traditional regularization the two contours shrink independently. On the other hand, using the proposed regularization, the parts of the contours that are close to each after few iterations snap onto each other

where

$$
\begin{aligned}
e_{a K}(\mathbf{x})= & \sum_{i=1}^{K} H_{\epsilon}\left(\phi_{i}(\mathbf{x})\right) \\
& +(-2)^{1} \sum_{i_{1}=1}^{K-1} \sum_{i_{2}=i_{1}+1}^{K} H_{\epsilon}\left(\phi_{i_{1}}(\mathbf{x})\right) H_{\epsilon}\left(\phi_{i_{2}}(\mathbf{x})\right) \\
& +(-2)^{2} \sum_{i_{1}=1}^{K-2} \sum_{i_{2}=i_{1}+1}^{K-1} \sum_{i_{3}=i_{2}+1}^{K} H_{\epsilon}\left(\phi_{i_{1}}(\mathbf{x})\right) \\
& \times H_{\epsilon}\left(\phi_{i_{2}}(\mathbf{x})\right) H_{\epsilon}\left(\phi_{i_{3}}(\mathbf{x})\right) \\
& +\cdots \\
& +(-2)^{K-2} \sum_{i_{1}=1}^{2} \ldots \sum_{i_{K}=i_{K-1}+1}^{K} H_{\epsilon}\left(\phi_{i_{1}}(\mathbf{x})\right) \ldots \\
& \times H_{\epsilon}\left(\phi_{i_{K}}(\mathbf{x})\right) \\
& +(-2)^{K-1} H_{\epsilon}\left(\phi_{i_{1}}(\mathbf{x})\right) \ldots H_{\epsilon}\left(\phi_{i_{K}}(\mathbf{x})\right)
\end{aligned}
$$

In the following we show that this term takes value 1 , if $\mathbf{x}$ belongs to a region characterized by an odd number of positive level set functions, and 0 elsewhere. In this way half of the partitions is penalized and the other half is favored (providing a principled extension of the two regions (i.e. one phase) case, in which the region where $\phi$ is positive is penalized and the complementary region is therefore favored). This will ensure that, as in the case of the 4 regions term, Properties 1 and 2 are always satisfied.

Lemma 3 For every point $\mathbf{x} \in \Omega$, let $K_{0}$ be the number of level set functions such that $\phi_{i}(\mathbf{x})>0$, or equivalently
$H_{\epsilon}\left(\phi_{i}(\mathbf{x})\right)=1$. We then have:

$e_{a K}(\mathbf{x})= \begin{cases}1 & \text { if } K_{0} \text { is odd } \\ 0 & \text { if } K_{0} \text { is even }\end{cases}$

Proof Considering that $H_{\epsilon}\left(\phi_{i}(\mathbf{x})\right)$ is approximately either 1 or 0 , we can rewrite (32) as:

$$
\begin{aligned}
e_{a K}(\mathbf{x})= & \left(\begin{array}{c}
K_{0} \\
1
\end{array}\right)+(-2)^{1}\left(\begin{array}{c}
K_{0} \\
2
\end{array}\right)+(-2)^{2}\left(\begin{array}{c}
K_{0} \\
3
\end{array}\right) \\
& +\cdots \\
& +(-2)^{K-2}\left(\begin{array}{c}
K_{0} \\
K-1
\end{array}\right)+(-2)^{K-1}\left(\begin{array}{c}
K_{0} \\
K
\end{array}\right)
\end{aligned}
$$

or in a more compact way:

$$
\begin{aligned}
e_{a K}(\mathbf{x}) & =\sum_{k=1}^{K}(-2)^{k-1}\left(\begin{array}{c}
K_{0} \\
k
\end{array}\right) \\
& =\sum_{k=0}^{K_{0}}(-2)^{k-1}\left(\begin{array}{c}
K_{0} \\
k
\end{array}\right)+\frac{1}{2}
\end{aligned}
$$

Now, rewriting the basic property of the binomial coefficient in (19) as:

$\frac{(x+y)^{K_{0}}}{y}=\sum_{k=0}^{K_{0}}\left(\begin{array}{c}K_{0} \\ k\end{array}\right) x^{n-k} y^{k-1}$

and choosing $x=1$ and $y=-2$ yields:

$$
-\frac{(-1)^{K_{0}}}{2}=\sum_{k=0}^{K_{0}}(-2)^{k-1}\left(\begin{array}{c}
K_{0} \\
k
\end{array}\right)
$$

Combining (35) and (37) we obtain:

$e_{a K}(\mathbf{x})=\frac{1-(-1)^{K_{0}}}{2}$

which concludes the proof.

Minimizing (31) w.r.t. $\phi_{1}$ (the derivation the other $\phi_{i}$, with $i=2, \ldots, K$, is similar) using calculus of variations and parameterizing the descent via the variable $t$, we obtain the following evolution equation for $\phi_{1}$ :

$$
\begin{aligned}
& \frac{\partial \phi_{1}(\mathbf{x})}{\partial t} \\
& =-\delta_{\epsilon}\left(\phi_{1}(\mathbf{x})\right)\left[1+(-2)^{1} \sum_{i_{2}=2}^{K} H_{\epsilon}\left(\phi_{i_{2}}(\mathbf{x})\right)\right. \\
& \quad+(-2)^{2} \sum_{i_{2}=2}^{K-1} \sum_{i_{3}=i_{2}+1}^{K} H_{\epsilon}\left(\phi_{i_{2}}(\mathbf{x})\right) H_{\epsilon}\left(\phi_{i_{3}}(\mathbf{x})\right)
\end{aligned}
$$




$$
\begin{aligned}
& +\cdots \\
& +(-2)^{K-2} \sum_{i_{2}=2}^{3} \ldots \sum_{i_{K}=i_{K-1}+1}^{K} H_{\epsilon}\left(\phi_{i_{2}}(\mathbf{x})\right) \ldots \\
& \times H_{\epsilon}\left(\phi_{i_{K}}(\mathbf{x})\right) \\
& \left.+(-2)^{K-1} H_{\epsilon}\left(\phi_{i_{2}}(\mathbf{x})\right) \ldots H_{\epsilon}\left(\phi_{i_{K}}(\mathbf{x})\right)\right]
\end{aligned}
$$

The following Lemma shows that for every point $\mathbf{x}$ such that $\phi_{i}(\mathbf{x})=0$ for at least one of the $i=2, \ldots, K$, then the term within the square brackets goes to zero (we assume as usual that $\left.H_{\epsilon}(0)=1 / 2\right)$. This guarantees that Property 1 holds true.

Lemma 4 Let $K \in \mathbb{N}$ and let $y \in \mathbb{R}^{K}$. Moreover, let $y_{i}$ denote the $i$-th component of $y$. Define the quantity

$$
\begin{aligned}
f_{K}(\mathbf{y})= & +(-2)^{1} \sum_{i_{2}=2}^{K} y_{i_{2}}+(-2)^{2} \sum_{i_{2}=2}^{K-1} \sum_{i_{3}=i_{2}+1}^{K} y_{i_{2}} y_{i_{3}} \\
& +\cdots \\
& +(-2)^{K-2} \sum_{i_{2}=2}^{3} \ldots \sum_{i_{K}=i_{K-1}+1}^{K} y_{i_{2}} \ldots y_{i_{K}} \\
& +(-2)^{K-1} y_{2} \ldots y_{K}
\end{aligned}
$$

assume w.l.o.g. that $y_{K}=1 / 2$ (the function is invariant to permutations of the components of $\mathbf{y}$ ). Then

$f_{K}(\mathbf{y})=0$

Proof Reasoning along the lines of Lemma 2 we can rewrite $f_{K}(\mathbf{y})$ as:

$f_{K}(\mathbf{y})=\left(1-2 y_{K}\right) f_{K-1}(\mathbf{y})$

Since $y_{K}=1 / 2$ by hypothesis, the proof is complete.

Summarizing this Section, we have introduced two new formulations for the length and area regularization terms. We have demonstrated that their combined effect satisfies the two desirable properties listed at the beginning of the section. In Fig. 7 we pictorially show the effect of the combination of these two regularization terms on the evolution of two interacting contours.

\section{Experimental Evaluation}

In this section, we provide both a qualitative and a quantitative comparison of the performance of the proposed regularization techniques with traditional multiphase regularization

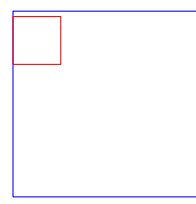

(a)

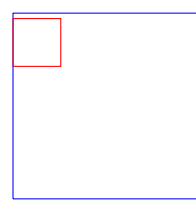

(e)

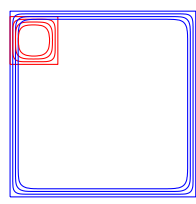

(b)

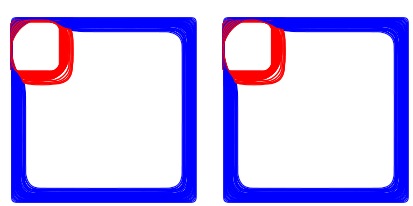

(f)

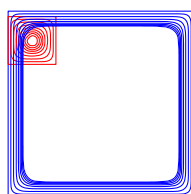

(c)

$(\mathrm{g})$

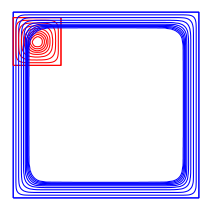

(d)

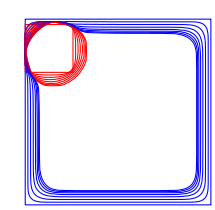

(h)
Fig. 7 First row: Evolution using the traditional length and area terms (Vese and Chan 2002), (a) Initial configuration, (b, c) Two stages of the evolution. Second row: Evolution using the proposed length and area terms, (e) Initial configuration, (f, g) Two stages of the evolution

techniques. To achieve this, we compare segmentation results obtained using the same data term (i.e. $E_{d}$ in equation (1)) but changing the two regularization terms ( $E_{l}$ and $\left.E_{a}\right)$. As a data term to drive the segmentation, we chose to use the popular Chan Vese term (Chan and Vese 2001, 2002), which aims to minimize the variance of the partitions.

In order to obtain a quantitative evaluation of the segmentation results, we compared the performance of our algorithm on the Berkeley Segmentation Data Set (BSDS) benchmark. The BSDS is composed of 100 images and for every image several human segmentations are provided. These human segmentations are considered ground truth and are used to compute precision $(p)$ and recall $(r)$ as measures of the accuracy of the segmentation. Precision is the probability that a pixel indicated as a boundary pixel by the segmentation algorithm is truly a boundary pixel. Recall is the probability that a true boundary pixel in the ground truth is correctly detected by the algorithm. Then the $F$-measure, i.e. the harmonic mean of precision and recall $\left(F=\frac{2 p r}{p+r}\right)$, is presented as a measure of performance.

In Fig. 8, we compare the F-measures obtained by the two different regularization techniques using the 4-region (i.e. two level set functions) segmentation model. The results are obtained by varying $\mu$ and $\nu$, respectively the coefficients of the length and area term as in (1). From the plots we can make the following important observations. First, the peak in the overall performance is reached using the proposed regularization technique. Second, we observe that the area term $E_{a}$, which uses the traditional regularization approach, was only detrimental to the overall segmentation (notice how the performance keeps dropping as the coefficient of the area term $v$ is increased). On the other hand, it becomes a useful regularizing contribution to the segmentation in our proposed regularization approach since the best scores are actually achieved choosing $v=1$. Third, the results are in general less sensitive to parameter tuning 


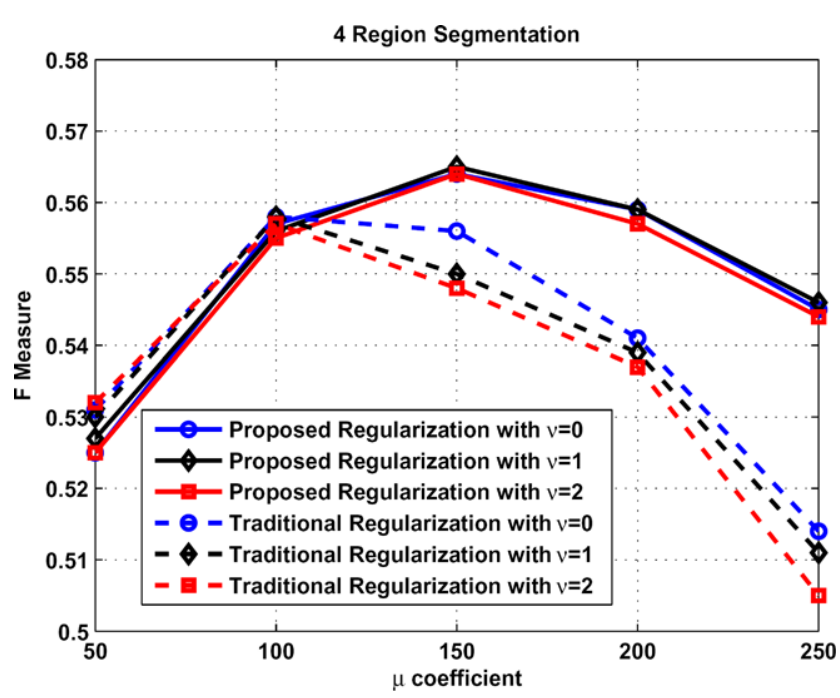

Fig. 8 F-measure plots comparing the proposed regularization schemes with the traditional ones for the 4-region segmentation model. The coefficient $\mu$ is the weight of the length term $E_{l}$, while $v$ is the weight of the area term $E_{a}$

using the proposed regularization since the performance decreases more slowly from the peak if the optimal parameter configuration is altered. This confirms the intuition that the proposed formulation, being more principled, is more robust than the traditional one.

Figure 9, which compares the F-measures obtained using the 8-region (i.e. three level set functions) segmentation model, is less informative than the previous one since the 8-region model is often too redundant for segmenting the images in the BSDS (as evidenced by the experiments presented in Bertelli et al. (2008)) and therefore the performance is lower than the one obtained using the 4-region model. Nonetheless we can still observe the same type of behavior manifested by the 4-region experiments, in the sense that the proposed regularization schemes outperform the traditional regularization on a wide range of parameter configurations. Also notice how the F-measure plots slowly decay using the proposed method, in contrast with a more pronounced drop for the traditional regularization methods, demonstrating the inherent robustness of our approach.

Figure 10 illustrates how critical the choice of effective multiphase regularization is in the case of volumetric segmentation. In this example we show the segmentation of a three-dimensional CT scan in 4 regions (bone, muscle, fat and air, see Fig. 10(a)). ${ }^{3}$ In Fig. 10(b) the 3D model of phase 1 (i.e. the rib cage) demonstrates that, using the traditional length and area regularization terms, it is not possible to remove many spurious isolated pixels. These noisy pixels are generated by two zero level set surfaces running almost

\footnotetext{
${ }^{3}$ High resolution versions of these results are available at: http://vision. ece.ucsb.edu/ lbertelli/research.html.
}

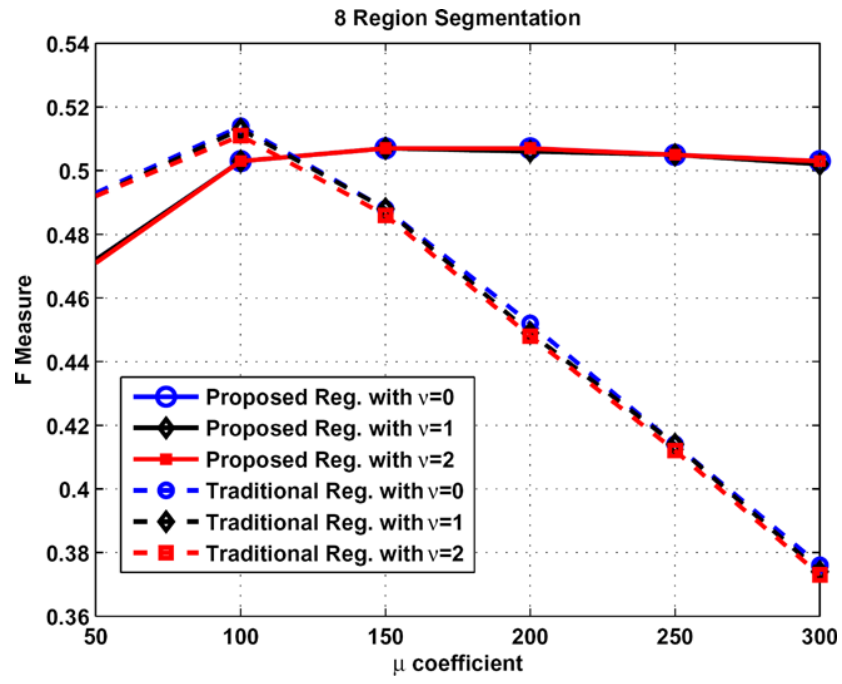

Fig. 9 F-measure plots comparing the proposed regularization schemes against the traditional ones for the 8-region segmentation model. The coefficient $\mu$ is the weight of the length term $E_{l}$, while $v$ is the weight of the area term $E_{a}$

parallel to each other locally. Since nothing is constraining or forcing the surfaces to stick to each other and given that these surfaces are already sufficiently smooth, the traditional regularization terms, which smooths or shrinks each surface independently, are not able to get rid of these spurious pixels. On the other hand, Fig. 10(c) illustrates how, after only 10 iterations of the proposed regularization, these noisy regions are completely eliminated.

In the last example (Fig. 11) we visually compare the effect of the two regularization flows on the same image segmentation task. In particular, we are interested in inspecting the effect of the area term $E_{a}$, which, in the case of the proposed scheme, enforces the validity of Property 2 described at the beginning of Sect. 3. The image to be segmented in 4 regions is the one shown in Fig. 11(a). Figure 11(b) shows the situation in terms of the level set functions after 500 iterations using the traditional regularization terms with $\mu=100$ and $\nu=1$. The green edge is the zero level set of $\phi_{1}$ and the red one is the zero level set of $\phi_{2}$. Notice how double edges are clearly visible all around the wings of the butterfly, demonstrating how the traditional scheme is not able to cope with these situations. We now want to show what happens if the area term weight $v$ is significantly increased. Figure 11(c,d) show the final segmentation using proposed and traditional area regularization terms respectively, starting from the configuration in Fig. 11(b). Using the proposed area term, double edges disappeared completely (Fig. 11(c)). On the other hand, the detrimental effect of the traditional area regularization term is shown in Fig. 11(d), where some double edges are removed, but at the expense of desirable edges, as depicted in the green circles. In addition, notice how some double edges are still 


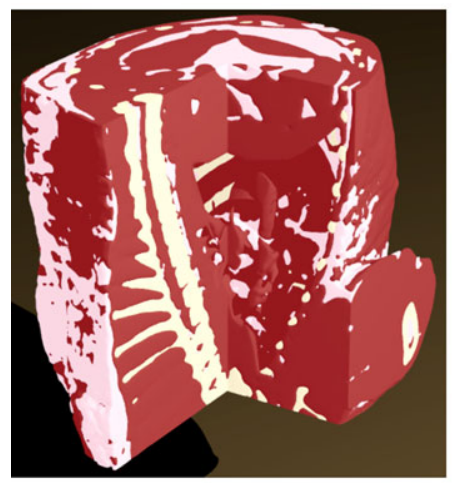

(a)

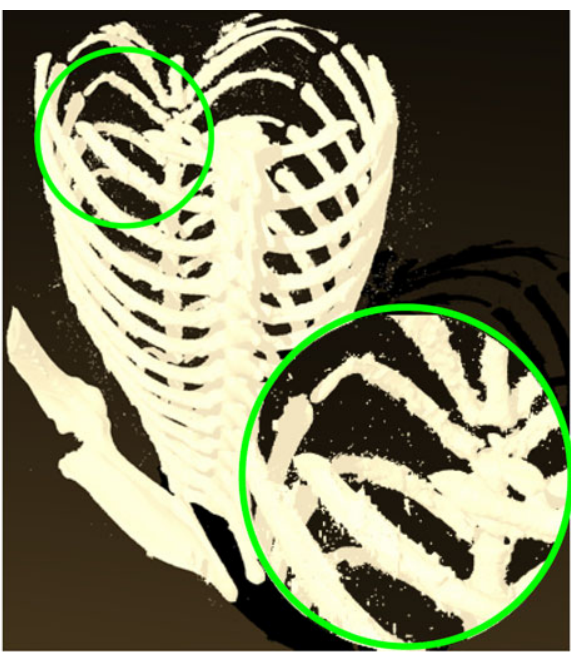

(b)

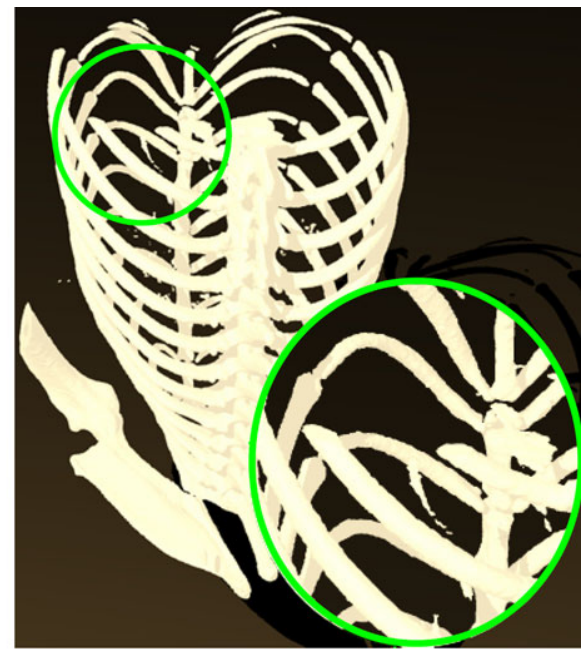

(c)
Fig. 10 (a) Segmentation of a volumetric CT scan in 4 regions (bone, muscle, fat and air). (b) Segmentation using the traditional length and area regularization terms. The zoom depicts the presence of many spurious isolated pixels that cannot be eliminated with this approach. (c) These artifacts are completely eliminated after applying only 10 iterations of the proposed regularization techniques

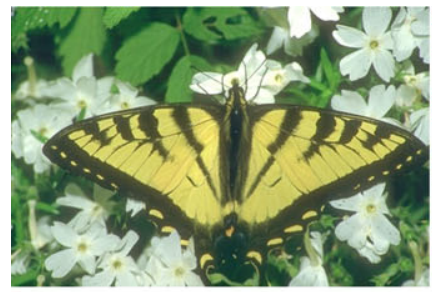

(a)



(e)



(i)

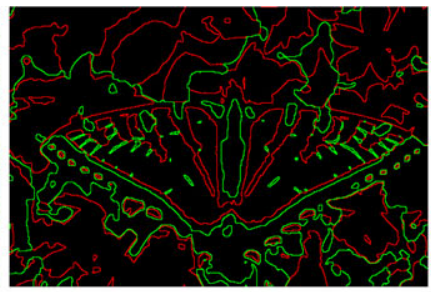

(b)

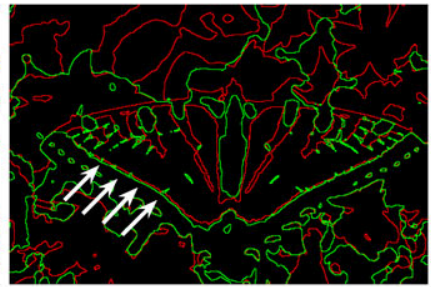

(f)

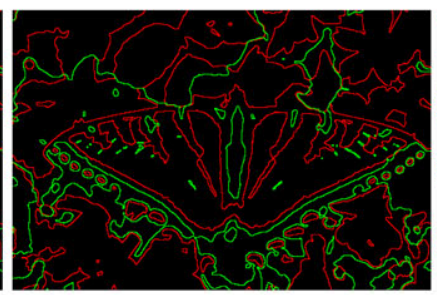

(j)

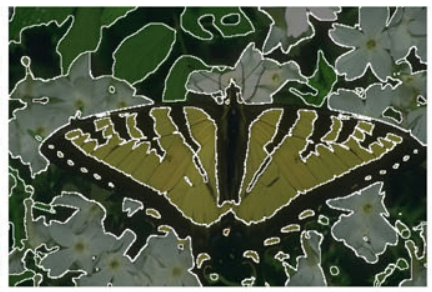

(c)



(g)

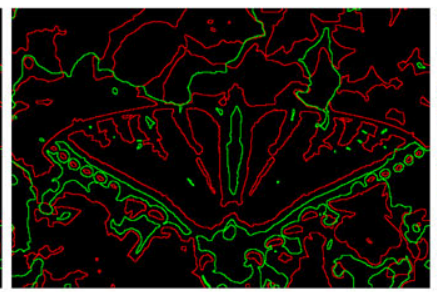

(k)

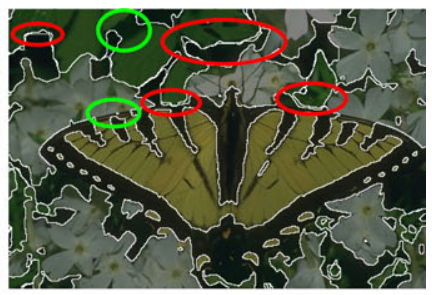

(d)

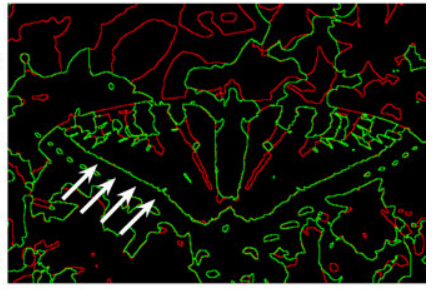

(h)

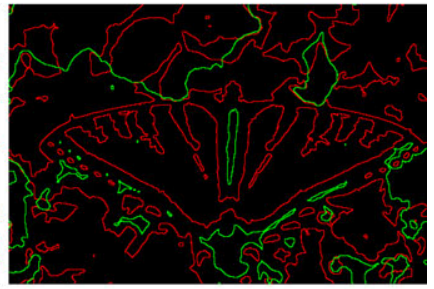

(1)

Fig. 11 (Color online) (a) Original image. (b) Situation after 500 iterations using the traditional regularization terms with $\mu=100$ and $v=1$. The green edge is the zero level set of $\phi_{1}$ and the red one is the zero level set of $\phi_{2}$. Double edges are clearly visible all around the wings of the butterfly. (c) Steady state solution after setting $v=160$ using the proposed regularization scheme, starting from the configuration in (b). Double edges are completely disappeared. (d) Steady state solution after setting $v=160$ using the traditional regularization scheme,

starting from the configuration in (b). Areas with the presence of double edges are highlighted in red, while areas with missing edges are circled in green. (e, $\mathbf{f}, \mathbf{g}, \mathbf{h})$ Snapshots of the evolution using the proposed regularization. Edges close to each other sense their reciprocal presence and they evolve accordingly (in this case snapping onto each other, see edges pointed by the arrows). (i, j, k, l) Snapshots of the evolution using the traditional regularization. Contours shrink regardless of their reciprocal position 
present regardless of how large the area coefficient is (see red circles). Finally snapshots of the evolution presented in the bottom two rows exemplify the importance of guaranteeing Properties 1 and 2: With the proposed regularization, contours sense each other and are able to snap onto each other, removing spurious regions. On the other hand, contours shrink irrespectively of their reciprocal position under the traditional regularization process, which can have detrimental effects as shown by the final segmentation result in Fig. 11(d).

\section{Conclusion}

In this paper we presented effective regularization techniques for multiphase level set based image segmentation. In particular, we reformulated the traditional length term to penalize the effective length of the multiphase contour and the area term to penalize half of the existing partitions. Mathematically this yields regularization equations in which the different level set functions involved in the segmentation process are coupled to each other, guaranteeing that the evolution of one of them affects the evolution of the others. In turn, this guarantees that Properties 1 and 2 are enforced. This is in contrast with traditional approaches, where this coupling is granted only by the data term (i.e. the term driving the segmentation), not in the regularization term. We qualitatively and quantitatively demonstrated that the segmentation results obtained using the proposed regularization technique outperforms the ones obtained via the traditional regularization.

Open Access This article is distributed under the terms of the Creative Commons Attribution Noncommercial License which permits any noncommercial use, distribution, and reproduction in any medium, provided the original author(s) and source are credited.

\section{Appendix A: Existence of Minimizers}

We now want to formally prove the existence of minimizers for the proposed cost functional, when characteristic functions are used in place of their mollified regularizers. We now restrict our attention, without loss of generality, to the two phases ( 4 regions) case. The $N$ regions scenario can be treated in a similar fashion. The functional $F\left(\chi_{E_{1}}, \chi_{E_{2}}\right)$ : $B V(\Omega) \times B V(\Omega) \mapsto \mathbb{R}$ that we are trying to minimize can be written as

$$
\begin{aligned}
\mathcal{F}\left(\chi_{E_{1}}, \chi_{E_{2}}\right)= & \mathcal{D}\left(\chi_{E_{1}}, \chi_{E_{2}}\right)+\mu \mathcal{L}\left(\chi_{E_{1}}, \chi_{E_{2}}\right) \\
& +v \mathcal{A}\left(\chi_{E_{1}}, \chi_{E_{2}}\right)
\end{aligned}
$$

where $\chi_{E_{1}}: \Omega \mapsto \mathbb{R}$ and $\chi_{E_{2}}: \Omega \mapsto \mathbb{R}$ are the characteristic functions of the sets $E_{1}$ and $E_{2}$ of finite perimeter, which therefore belong to the space of bounded variations $B V(\Omega)$. $\mathcal{D}\left(\chi_{E_{1}}, \chi_{E_{2}}\right)$ is the data term, defined along the lines of Chan and Vese (2001) as

$$
\begin{aligned}
\mathcal{D}\left(\chi_{E_{1}}, \chi_{E_{2}}\right)= & \int_{\Omega}\left(u_{0}-c_{1}\right)^{2} \chi_{E_{1}} \chi_{E_{2}} \\
& +\int_{\Omega}\left(u_{0}-c_{2}\right)^{2} \chi_{E_{1}}\left(1-\chi_{E_{2}}\right) \\
& +\int_{\Omega}\left(u_{0}-c_{3}\right)^{2}\left(1-\chi_{E_{1}}\right) \chi_{E_{2}} \\
& +\int_{\Omega}\left(u_{0}-c_{4}\right)^{2}\left(1-\chi_{E_{1}}\right)\left(1-\chi_{E_{2}}\right)
\end{aligned}
$$

In order to define $\mathcal{L}\left(\chi_{E_{1}}, \chi_{E_{2}}\right)$, we introduce $\partial E_{1}$ as the perimeter of set $E_{1}$ and $\Pi\left(\partial E_{1}\right)$ as the measure of the perimeter. We can then write our proposed length term as

$\mathcal{L}\left(\chi_{E_{1}}, \chi_{E_{2}}\right)=\Pi\left(\partial E_{1}\right)+\Pi\left(\partial E_{2}\right)-\Pi\left(\partial E_{1} \cap \partial E_{2}\right)$

Similarly, in order to define $\mathcal{A}\left(\chi_{E_{1}}, \chi_{E_{2}}\right)$ we introduce $\Lambda\left(E_{1}\right)$ as the measure of the area of set $E_{1}$. The proposed area term becomes then

$\mathcal{A}\left(\chi_{E_{1}}, \chi_{E_{2}}\right)=\Lambda\left(E_{1}\right)+\Lambda\left(E_{2}\right)-2 \Lambda\left(E_{1} \cap E_{2}\right)$

In proving the existence of the minimizer we proceed along the lines of Chan and Vese (1998), using the following basic result regarding bounded variation spaces (Evans and Gariepy 1992) (other similar ideas can be found in Chung and Vese 2009).

Result 1 Consider the Banach space BV $(\Omega)$ endowed with the norm

$\|u\|_{B V(\Omega)}=\|u\|_{L^{1}(\Omega)}+|D u|(\Omega)$

If $\left(u^{n}\right)_{n \geq 1}$ is a bounded sequence in $B V(\Omega)$, then there exists a subsequence $\left(u^{n_{j}}\right)$ of $\left(u^{n}\right)$ and a function $u \in B V(\Omega)$, such that $u^{n_{j}} \rightarrow u$ strongly in $L^{1}(\Omega)$ as $n_{j} \rightarrow \infty$, and

$|D u| \leq \lim _{n_{j} \rightarrow \infty} \inf \left|D u^{n_{j}}\right|$

If $u$ and $\left(u^{n}\right)$ are characteristic functions, i.e. $u=\chi_{E}$ and $\left(u^{n}\right)=\left(\chi_{E}^{n}\right)$, then (47) reads in terms of perimeters as

$\Pi\left(\partial \chi_{E}\right) \leq \lim _{n_{j} \rightarrow \infty} \inf \Pi\left(\partial \chi_{E}^{n_{j}}\right)$

We now prove the existence of the minimizer:

Theorem 1 If $u_{0} \in L^{\infty}(\Omega)^{4}$, then the following minimization problem

$$
\begin{aligned}
& \inf _{\chi_{E_{1}}, \chi_{E_{2}}} F\left(\chi_{E_{1}}, \chi_{E_{2}}\right), \quad\left[\chi_{E_{1}}, \chi_{E_{2}}\right] \in B V(\Omega) \times B V(\Omega) \\
& \quad \chi_{E_{1}} \in\{0,1\} d x \text {-a.e., } \chi_{E_{2}} \in\{0,1\} d x \text {-a.e. }
\end{aligned}
$$

has a solution.

${ }^{4}$ We can assume, without loss of generality, that the original image $u_{0}$ is bounded, since it is the result of a sensor acquisition and therefore its values are bound to be in the sensor range. 
Proof Let $\left(\left[\chi_{E_{1}}^{n}, \chi_{E_{2}}^{n}\right]\right)_{n \geq 1}$ be a minimizing sequence of $\mathcal{F}$, i.e.

$\inf _{\chi_{E_{1}}, \chi_{E_{2}}} \mathcal{F}\left(\chi_{E_{1}}, \chi_{E_{2}}\right)=\lim _{n \rightarrow \infty} \mathcal{F}\left(\chi_{E_{1}}^{n}, \chi_{E_{2}}^{n}\right)$

Then there exist a constant $M>0$ such that (we can see in fact that by choosing $\chi_{E_{1}}^{n} \equiv 1$ and $\chi_{E_{2}}^{n} \equiv 1$ the functional is bounded by $\left.\left\|u_{0}\right\|_{L^{2}(\Omega)}\right)$

$\mathcal{F}\left(\chi_{E_{1}}^{n}, \chi_{E_{2}}^{n}\right) \leq M$

By making use of (42), where all three term are positive, we can also write

$\mathcal{L}\left(\chi_{E_{1}}^{n}, \chi_{E_{2}}^{n}\right) \leq M$

By construction, see (44), $\mathcal{L}\left(\chi_{E_{1}}^{n}, \chi_{E_{2}}^{n}\right)$ is also bounded from below by:

$\max \left(\Pi\left(E_{1}{ }^{n}\right), \Pi\left(E_{2}^{n}\right)\right) \leq \mathcal{L}\left(\chi_{E_{1}}^{n}, \chi_{E_{2}}^{n}\right)$

which leads to the desired bound on the two measures of perimeters

$\max \left(\Pi\left(E_{1}^{n}\right), \Pi\left(E_{2}^{n}\right)\right) \leq M$

The two areas are bounded by the area of the domain, therefore we can write

$$
\begin{aligned}
& \max \left(\Lambda\left(E_{1}^{n}\right), \Lambda\left(E_{2}^{n}\right)\right) \\
& \quad=\max \left(\left\|\chi_{E_{1}}^{n}\right\|_{L^{1}(\Omega)},\left\|\chi_{E_{2}}^{n}\right\|_{L^{1}(\Omega)}\right) \leq|\Omega|
\end{aligned}
$$

We demonstrated that the sequence $\left[\chi_{E_{1}}^{n}, \chi_{E_{2}}^{n}\right] \in B V(\Omega) \times$ $B V(\Omega)$ and therefore, by virtue of Result 1 , we have the existence of a minimizer $\left[\chi_{E_{1}}, \chi_{E_{2}}\right] \in B V(\Omega) \times B V(\Omega)$. Hence $\chi_{E_{1}}$ and $\chi_{E_{2}}$ are two minimizers of $\mathcal{F}$ in the space of characteristic functions of finite perimeter.

\section{Appendix B: Behavior in the Limit for $\epsilon \rightarrow 0$}

We now want to formally prove that for $\epsilon \rightarrow 0$ the length term of the cost functional $\left(\mathcal{L}\left(\chi_{E_{1}}, \chi_{E_{2}}\right)\right)$ becomes the measure (in a measure theoretic sense) of the contour of the multi-phase level set model, as claimed in the paper. First of all, we formulate the length cost functional in (44) in terms of the two level set functions ( $\phi_{1}$ and $\left.\phi_{2}\right)$ and by using mollified Heaviside functions. We will describe a generic mollified $H_{\epsilon}$ as the convolution between $H$ and a mollifier $\eta_{\epsilon}$ (positive, even and monotonically decreasing away from 0$),{ }^{5}$ where $\epsilon$ is the parameter that regulates the smooth-

\footnotetext{
${ }^{5}$ Note that these assumptions have been made to simplify the notation of this proof, but similar considerations can be extended to other types of mollifiers as well.
}

ness of the mollifier $\left(\lim _{\epsilon \rightarrow 0} \eta_{\epsilon}=\delta\right)$. Hence we can write

$$
\begin{aligned}
\mathcal{L}_{\epsilon}\left(\phi_{1}, \phi_{2}\right) & \\
= & \int_{\Omega}\left|\nabla\left(\left(\eta_{\epsilon} * H\right)\left(\phi_{1}\right)\right)\right|+\int_{\Omega}\left|\nabla\left(\left(\eta_{\epsilon} * H\right)\left(\phi_{2}\right)\right)\right| \\
& -\int_{\Omega} \gamma\left|\nabla\left(\left(\eta_{\epsilon} * H\right)\left(\phi_{1}\right)\right)\right|\left|\nabla\left(\left(\eta_{\epsilon} * H\right)\left(\phi_{2}\right)\right)\right|
\end{aligned}
$$

where $\gamma$ is chosen as a normalizing factor such that the function

$\mathcal{C}_{\epsilon}\left(\phi_{2}(x, y)\right)=\gamma\left|\nabla\left(\left(\eta_{\epsilon} * H\right)\left(\phi_{2}(x, y)\right)\right)\right|$

attains value 1 on the zero level set of $\phi_{2}$, that is

$\mathcal{C}_{\epsilon}\left(\phi_{2}(x, y)\right)=1 \quad$ if $\phi_{2}(x, y)=0$

In this way, in the limit, the function

$\chi_{\partial E_{2}}=\lim _{\epsilon \rightarrow 0} \mathcal{C}_{\epsilon}=\lim _{\epsilon \rightarrow 0} \gamma\left|\nabla\left(\eta_{\epsilon} * \chi_{E_{2}}\right)\right|$

becomes the indicator function for the boundary of the set $E_{2}$ (i.e. the subset of $\Omega$, where $\phi_{2}=0$ ). ${ }^{6}$ Now we can use the results in Evans and Gariepy (1992) to show that in the limit

$$
\begin{aligned}
\lim _{\epsilon \rightarrow 0} \mathcal{L}_{\epsilon}\left(\phi_{1}, \phi_{2}\right)= & \int_{\Omega}\left|\nabla H\left(\phi_{1}\right)\right|+\int_{\Omega}\left|\nabla H\left(\phi_{2}\right)\right| \\
& -\int_{\Omega \cap \partial E_{2}}\left|\nabla H\left(\phi_{1}\right)\right|
\end{aligned}
$$

We can notice in fact how the same measure theoretical properties (demonstrated in Evans and Gariepy 1992 and exploited by Chan and Vese 1998, 2001) that apply to the first two integrals apply also to the last one, which is simply differing from the first two in that it is computed on a subset of the domain $\Omega$.

We now prove that the method is stable for $\epsilon \rightarrow 0$. We will make use of the same compactness results that was used to prove the existence of minimizers. We would like to point out that this proof was not present in Chan and Vese (1998, 2001), but we feel it can be important to complete the argument.

Define

$h_{1}^{\epsilon}=\left(\eta_{\epsilon} * H\right)\left(\phi_{1}\right) \quad$ and $\quad h_{2}^{\epsilon}=\left(\eta_{\epsilon} * H\right)\left(\phi_{2}\right)$

${ }^{6}$ If we assume $|\nabla \phi|=1$, then $\gamma$ becomes a constant and its explicit expression can be written as

$\gamma=\frac{1}{\left(\eta_{\epsilon} * H\right)^{\prime}(0)}$

We would like to point out the choice of $\gamma=1 / \delta_{\epsilon}(0)$, made in the paper, is a particular case of (60). 
We can now write the mollified versions of (43), (44) and (45) in terms of $h_{1}^{\epsilon}$ and $h_{2}^{\epsilon}$.

$$
\begin{aligned}
& \mathcal{D}_{\epsilon}\left(h_{1}^{\epsilon}, h_{2}^{\epsilon}\right) \\
& =\int_{\Omega}\left(u_{0}-c_{1}\right)^{2} h_{1}^{\epsilon} h_{2}^{\epsilon}+\int_{\Omega}\left(u_{0}-c_{2}\right)^{2} h_{1}^{\epsilon}\left(1-h_{2}^{\epsilon}\right) \\
& \quad+\int_{\Omega}\left(u_{0}-c_{3}\right)^{2}\left(1-h_{1}^{\epsilon}\right) h_{2}^{\epsilon} \\
& \quad+\int_{\Omega}\left(u_{0}-c_{4}\right)^{2}\left(1-h_{1}^{\epsilon}\right)\left(1-h_{2}^{\epsilon}\right) \\
& \mathcal{L}_{\epsilon}\left(h_{1}^{\epsilon}, h_{2}^{\epsilon}\right)=\int_{\Omega}\left|\nabla h_{1}^{\epsilon}\right|+\int_{\Omega}\left|\nabla h_{2}^{\epsilon}\right|-\int_{\Omega} \gamma\left|\nabla h_{1}^{\epsilon}\right|\left|\nabla h_{2}^{\epsilon}\right| \\
& \mathcal{A}_{\epsilon}\left(h_{1}^{\epsilon}, h_{2}^{\epsilon}\right)=\int_{\Omega} h_{1}^{\epsilon}+\int_{\Omega} h_{2}^{\epsilon}-2 \int_{\Omega} h_{1}^{\epsilon} h_{2}^{\epsilon}
\end{aligned}
$$

Therefore

$$
\mathcal{F}_{\epsilon}\left(h_{1}^{\epsilon}, h_{2}^{\epsilon}\right)=\mathcal{D}_{\epsilon}\left(h_{1}^{\epsilon}, h_{2}^{\epsilon}\right)+\mathcal{L}_{\epsilon}\left(h_{1}^{\epsilon}, h_{2}^{\epsilon}\right)+\mathcal{A}_{\epsilon}\left(h_{1}^{\epsilon}, h_{2}^{\epsilon}\right)
$$

Finally let

$$
\left[g_{1}^{\epsilon}, g_{2}^{\epsilon}\right]=\arg \inf _{h_{1}^{\epsilon}, h_{2}^{\epsilon}} \mathcal{F}_{\epsilon}\left(h_{1}^{\epsilon}, h_{2}^{\epsilon}\right)
$$

The existence of a minimizer for (67) can be demonstrated along the lines of Theorem 1 .

Theorem 2 The sequence $\left(\left[g_{1}^{1 / n}, g_{2}^{1 / n}\right]\right)_{n \geq 1} \in B V(\Omega) \times$ $B V(\Omega)$ admits a subsequence $\left(\left[g_{1}^{1 / n_{j}}, g_{2}^{1 / n_{j}}\right]\right)_{n \geq 1} \in$ $B V(\Omega) \times B V(\Omega)$ such that, there exists a vector $\left[g_{1}, g_{2}\right]$ bounded in $B V(\Omega) \times B V(\Omega)$ such that

$$
\left(\left[g_{1}^{1 / n_{j}}, g_{2}^{1 / n_{j}}\right]\right) \rightarrow\left[g_{1}, g_{2}\right]
$$

strongly in $L^{1}(\Omega)$ as $n_{j} \rightarrow \infty$,

$$
\left|D g_{1}\right| \leq \lim _{n_{j} \rightarrow \infty} \inf \left|D h_{1}^{1 / n_{j}}\right|
$$

and

$$
\left|D g_{2}\right| \leq \lim _{n_{j} \rightarrow \infty} \inf \left|D h_{2}^{1 / n_{j}}\right|
$$

Proof We need to show that the sequence $\left(\left[g_{1}^{1 / n}, g_{2}^{1 / n}\right]\right)_{n \geq 1}$ is bounded in $B V(\Omega) \times B V(\Omega)$ (a bound independent of $n$ ), then the proof follows by applying Result 1 .

We begin by noting that choosing $h_{1}^{1 / n} \equiv 1$ and $h_{2}^{1 / n} \equiv 1$ we have

$$
\mathcal{F}_{\epsilon}\left(h_{1}^{1 / n}, h_{2}^{1 / n}\right) \leq\left\|u_{0}\right\|_{L^{2}(\Omega)}=M
$$

since $\mathcal{L}_{\epsilon}=0$ and $\mathcal{A}_{\epsilon}=0$. Therefore, in light of (67), we have

$$
\mathcal{F}_{\epsilon}\left(g_{1}^{1 / n}, g_{2}^{1 / n}\right) \leq M
$$

from which we deduce bounds on the length and area terms as well (since all three terms in (66) are positive)

$$
\begin{aligned}
& \mathcal{L}_{\epsilon}\left(g_{1}^{1 / n}, g_{2}^{1 / n}\right) \leq M \\
& \mathcal{A}_{\epsilon}\left(g_{1}^{1 / n}, g_{2}^{1 / n}\right) \leq M
\end{aligned}
$$

Therefore we have

$$
\begin{aligned}
& \max \left(\left|D g_{1}^{1 / n}\right|(\Omega),\left|D g_{2}^{1 / n}\right|(\Omega)\right) \leq M \\
& \max \left(\left\|g_{1}^{1 / n}\right\|_{L^{1}(\Omega)},\left\|g_{2}^{1 / n}\right\|_{L^{1}(\Omega)}\right) \leq M
\end{aligned}
$$

which demonstrates that the sequence $\left(\left[g_{1}^{1 / n}, g_{2}^{1 / n}\right]\right)_{n \geq 1}$ is bounded in $B V(\Omega) \times B V(\Omega)$ and hence completes the proof.

Acknowledgements The authors would like to thank the reviewers for their constructive comments and Dr. Ruggero Carli for numerous fruitful discussions. This research was supported by NSF ITR grant \#0331697 and NSF award III-0808772.

\section{References}

Aubert, G., Barlaud, M., Faugeras, O., \& Jehan-Besson, S. (2003). Image segmentation using active contours: Calculus of variations or shape gradients? SIAM Applied Mathematics, 63, 2128-2154.

Bertelli, L., Sumengen, B., Manjunath, B., \& Gibou, F. (2008). A variational framework for multi-region pairwise similarity-based image segmentation. IEEE Transactions on Pattern Analysis and Machine Intelligence, 30(8), 1400-1414.

Caselles, V., Kimmel, R., \& Sapiro, G. (1995).Geodesic active contours. In IEEE international conference on computer vision (ICCV) (pp. 694-699).

Caselles, V., Kimmel, R., \& Sapiro, G. (1997). Geodesic active contours. International Journal of Computer Vision, 22(1), 61-80.

Chan, T. F., \& Vese, L. A. (1998). Active contours without edges. Tech. Rep. 98-53, UCLA, ftp://ftp.math.ucla.edu/pub/camreport/ cam98-53.ps.gz.

Chan, T. F., \& Vese, L. A. (2001). Active contours without edges. IEEE Transactions on Image Processing, 10(2), 266-277.

Chung, G., \& Vese, L. A. (2009). Image segmentation using a multilayer level-set approach. Computing and Visualization in Science, 12(6), 1634-1641.

Cremers, D. (2006). Dynamical statistical shape priors for level set based tracking. IEEE Transactions on Pattern Analysis and Machine Intelligence, 28(8), 1262-1273.

Cremers, D., Sochen, N., \& Schnorr, C. (2004). Multiphase dynamic labeling for variational recognition-driven image segmentation. In European conference on computer vision (ECCV) (pp. 74-86). Berlin: Springer.

Cremers, D., Osher, S. J., \& Soatto, S. (2006). Kernel density estimation and intrinsic alignment for shape priors in level set segmentation. International Journal of Computer Vision, 69(3), 335-351.

Dambreville, S., Rathi, Y., \& Tannenbaum, A. (2006). Shape-based approach to robust image segmentation using kernel PCA. In IEEE computer society conference on computer vision and pattern recognition (CVPR) (pp. 977-984).

Evans, L. C., \& Gariepy, R. F. (1992). Measure theory and fine properties of functions. Boca Raton: CRC Press.

Goldenberg, R., Kimmel, R., Rivlin, E., \& Rudzsky, M. (2001). Fast geodesic active contours. IEEE Transactions on Image Processing, 10, 1467-1475. 
Kass, M., Witkin, A., \& Terzopoulos, D. (1988). Snakes: active contour models. International Journal of Computer Vision, 1, 321-331.

Kichenassamy, S., Kumar, A., Olver, P., Tannenbaum, A., \& Yezzi, A. (1995). Gradient flows and geometric active contour models. In International conference on computer vision (ICCV) (pp. 810815).

Kim, J., Fisher, J., Cetin, M., \& Willsky, A. (2005). A nonparametric statistical method for image segmentation using information theory and curve evolution. IEEE Transactions of Image Processing, 14(10), 1486-1502.

Malladi, R., Sethian, J. A., \& Vemuri, B. C. (1994). Evolutionary fronts for topology-independent shape modeling and recovery. In European conference on computer vision (ECCV) (Vol. 1, pp. 3-13).

Malladi, R., Sethian, J. A., \& Vemuri, B. C. (2004). A topology independent shape modeling scheme. In SPIE conference on geometric methods in computer vision (pp. 246-258).

Mumford, D., \& Shah, J. (1985). Boundary detection by minimizing functionals. In IEEE computer society conference on computer vision and pattern recognition (CVPR) (pp. 22-26).

Nguyen, H. T., Worring, M., \& van den Boomgaard, R. (2003). Watersnakes: energy-driven watershed segmentation. IEEE Transactions on Pattern Analysis and Machine Intelligence, 25, 330-342.

Osher, S., \& Sethian, J. A. (1988). Fronts propagating with curvaturedependent speed: Algorithms based on Hamilton-Jacobi formulations. Journal of Computational Physics, 79, 12-49.

Paragios, N., \& Deriche, R. (1999). Geodesic active regions for supervised texture segmentation. In IEEE international conference on computer vision (ICCV) (Vol. 2, pp. 926-932).

Paragios, N., \& Deriche, R. (2002). Geodesic active regions and level set methods for supervised texture segmentation. International Journal of Computer Vision, 46, 223-247.

Park, J., \& Keller, J. (2001). Snakes on the watershed. IEEE Transactions on Pattern Analysis and Machine Intelligence, 23, 12011205.

Rathi, Y., \& Michailovich, O. (2006). Seeing the unseen: Segmenting with distributions. In International conference on signal and image processing.

Raviv, T. R., Kiryati, N., \& Sochen, N. (2005). Prior-based segmentation by projective registration and level sets. In IEEE International conf. on computer vision (ICCV) (pp. 204-211).

Raviv, T. R., Kiryati, N., \& Sochen, N. (2006). Prior-based segmentation and shape registration in the presence of projective distor- tion. International Journal of Computer Vision (IJCV). Accepted for publication.

Rousson, M., Brox, T., \& Deriche, R. (2003). Active unsupervised texture segmentation on a diffusion based feature space. In IEEE conference on computer vision and pattern recognition (CVPR) (Vol. 2, pp. 699-704).

Samson, C., Blanc-Feraud, L., Aubert, G., \& Zerubia, J. (1999). A level set model for image classification. In Proc. of scale-space theory in computer vision.

Samson, C., Blanc-Feraud, L., Aubert, G., \& Zerubia, J. (2000a). A variational model for image classification and restoration. IEEE Transactions on Pattern Analysis and Machine Intelligence, 22(5), 460-472.

Samson, C., Blanc-Feraud, L., Aubert, G., \& Zerubia, J. (2000b). A level set model for image classification. International Journal of Computer Vision, 40, 187-197.

Sapiro, G. (1996). Vector (self) snakes: a geometric framework for color, texture, and multiscale image segmentation. In IEEE international conference on image processing (ICIP) (pp. 817-820).

Sapiro, G. (1997). Color snakes object segmentation. Computer Vision and Image Understanding, 68, 247-253.

Tsai, A., Yezzi, A. J., \& Willsky, A. S. (2001). Curve evolution implementation of the Mumford-Shah functional for image segmentation, denoising, interpolation, and magnification. IEEE Transactions on Image Processing, 10, 1169-1186.

Vese, L. A. (2003). Multiphase object detection and image segmentation. In Geometric level set methods in imaging, vision and graphics (pp. 175-194). Berlin: Springer.

Vese, L. A., \& Chan, T. F. (2002). A multiphase level set framework for image segmentation using the Mumford and shah model. International Journal of Computer Vision, 50, 271-293.

Yezzi, A. J., Tsai, A., \& Willsky, A. (1999). A statistical approach to snakes for bimodal and trimodal imagery. In International conference on computer vision (ICCV) (pp. 898-903).

Zhu, S. C., \& Yuille, A. (1996). Region competition: unifying snakes, region growing, and bayes/mdl for multiband image segmentation. IEEE Transactions on Pattern Analysis and Machine Intelligence, 18, 884-900.

Zhu, S. C., Lee, T. S., \& Yuille, A. L. (1995). Region competition: unifying snakes, region growing, energy/bayes/mdl for multi-band image segmentation. In IEEE international conference on computer vision (ICCV) (pp. 416-423). 